Bio-grafía: Escritos sobre la Biologia y su Enseñanza Vol. 4 No 7. ISSN 2027-1034.

Segundo Semestre de 2011. P. p. 36-55

\title{
EL LENGUAJE CIENTÍFICO Y SU RELACIÓN CON LA COMPRENSIÓN DEL PROCESO DE EXCRECIÓN ANIMAL
}

\section{SCIENTIFIC LANGUAGE AND THE UNDERSTANDING OF ANIMAL EXCRETION PROCESS}

\author{
Por: Erika Lasprilla ${ }^{1}$; Análida Hernández ${ }^{2}$
}

\begin{tabular}{|l|}
\hline Recibido:24-06-2011 \\
\hline Aceptado:10-12-2011 \\
\hline
\end{tabular}

\section{Resumen}

Esta investigación consiste en el análisis del lenguaje científico y su relación con la comprensión del proceso de excreción animal, a través del uso de una guía de trabajo diagnóstica con estudiantes de grado séptimo del Centro Educativo Distrital Calasanz de Bogotá. Para el desarrollo del objetivo se caracteriza la población de estudio, identificando el grado de apropiación del lenguaje científico, así como, las principales causas que dificultan su comprensión. La investigación es de carácter cualitativo, emplea la interpretación por medio de la observación participante, los instrumentos son el diario de clase y la guía de trabajo diagnóstica, la cual permite evaluar la habilidad de los estudiantes para responder preguntas de tipo literal, inferencial, evaluativo y creativo.

De acuerdo con los resultados de la investigación, se sabe que la población de estudio responde mejor las preguntas evaluativas, seguido de literales, creativas e inferenciales. Concluyendo así que, la estimación del grado de apropiación de las características del lenguaje facilita el reconocimiento de fortalezas y debilidades de la comunicación de ideas científicas de los estudiantes. De la misma forma, las condiciones de vida, y por ende, de educación de la población de estudio están determinadas por factores como el estrato socio económico al cual pertenece, el bajo nivel académico de sus padres y la conformación del núcleo familiar. Por otro lado, una de las causas de dificultad en la comprensión del lenguaje científico es la falta de habilidad lectora de los alumnos.

PALABRAS CLAVE: Lenguaje científico, excreción animal, guía de trabajo diagnóstica preguntas literales, Inferenciales, evaluativas y creativas.

\section{ABSTRACT}

This research studies the relationship between scientific language and the understanding of the animal excretion process through the implementation of an

${ }^{1}$ Bióloga, Especialista - Docente del Centro Educativo Distrital Calasanz. E-mail: erikalasprilla@yahoo.com. Autora.

${ }^{2}$ Bióloga, Magister - Docente Universidad Pedagógica Nacional de Bogotá. E-mail: anaerpi@hotmail.com. Coautora. 
assessment guide to be used by seven grade students from the Centro Educativo Distrital Calasanz in Bogotá. To accomplish this goal, the experimental group is characterized identifying the degree of appropriation of the scientific language as well as the main causes that hinder its understanding. This qualitative research gathers data from the observation of participants, from the class journal and the assessment guide tool that evaluate the student skills in answering questions in verbatim, inferential, assessment or creative ways.

KEYWORDS: Scientific language, animal excretion process, assessment guide, questions in verbatim, inferential, assessment and creative ways.

\section{Introducción:}

Teniendo en cuenta la relevancia que tienen los procesos de comunicación en la enseñanza de la Biología, se realiza una investigación para mejorar la comprensión, la formación y la calidad educativa de las ciencias a partir del uso del lenguaje científico. Bajo esta concepción, Foucault (1983) plantea que la enseñanza no puede pensarse solamente como la transmisión de contenidos, sino que adquiere un sentido diferente: el de asignar, distribuir y adecuar los discursos a los sujetos y éstos a aquellos, a fin de hacer posible un pensamiento, para este caso, "el científico".

Desde otro enfoque, el lenguaje de las ciencias naturales es amplio, razón por la cual, no siempre se alcanza la comprensión esperada en el aprendizaje de la disciplina. Para Kempa (1991), los problemas de comunicación derivados del uso del lenguaje representan una de las principales causas de dificultad en el entendimiento de las ciencias. Partiendo de estas referencias, surge la necesidad de profundizar en el análisis de la percepción del lenguaje biológico empleado en el aula.

A partir de un ejercicio reflexivo sobre los procesos de enseñanza - aprendizaje, se analiza el uso del lenguaje científico en un grupo de 43 estudiantes de grado séptimo, con respecto al tema de excreción animal por medio de una guía de trabajo diagnóstica. El escenario seleccionado es el Centro Educativo Distrital Calasanz en la localidad de Ciudad

Bolívar -Bogotá D.C-

Por otra parte, el Centro Educativo Distrital Calasanz, es un colegio en concesión que cumple diez años y educa a 1203 estudiantes anualmente. Sin embargo, aunque se mantiene el número total de alumnos, algunos no culminan el grado y por lo tanto, se incorporan nuevos niños y niñas durante el año escolar, lo que generalmente causa dificultades académicas. Otro fenómeno social del contexto es la falta de acompañamiento familiar en la realización de tareas, desnutrición, abandono, violencia, etc., por estas y otras razones, como por ejemplo, el alto número de estudiantes por aula y la desmotivación escolar, el lenguaje empleado en las clases de Biología no posee el nivel esperado.

Con respecto a la metodología y a los referentes teóricos, se retoman y modifican algunos criterios sobre los procesos comunicativos de la enseñanza de las ciencias, considerando específicamente los aportes del artículo "Cómo Promover Distintos Niveles de Lectura de los Textos de Ciencias" escrito por Sardá, Márquez y Sanmartí (2006). En el documento, se presenta una estrategia de lectura basada en el planteamiento de preguntas. Como 
objetivo se propone promover distintos niveles de lecturas en textos científicos, partiendo de la hipótesis de que el tipo de preguntas que se hace sobre las lecturas puede ayudar a los estudiantes a desarrollar estrategias lectoras. Se analizan las respuestas de estudiantes adolescentes, acerca de cuestionarios sobre una lectura del libro de texto de química. Se incluyen cuatro estilos de preguntas: lectura literal (¿qué dice el texto?), lectura inferencial (¿qué informaciones no dice el texto, pero necesito saber para entenderlo?), lectura evaluativa (¿cuáles son las ideas más importantes?, ¿qué ideas aporta y que eran desconocidas para el estudiante?), lectura creativa (¿para qué sirve este texto?, ¿estas ideas pueden ser útiles para interpretar otros fenómenos?).

Para la presente investigación se parte de la temática de excreción animal, ya que usualmente el manejo de su vocabulario causa dificultades en los estudiantes y además, forma parte de los contenidos programados desde la Institución y los Estándares Básicos de competencias en Ciencias Naturales. En la guía de trabajo diagnóstica se formulan los cuatro estilos de interrogantes propuestos por Sardá et.al., (2006) en correspondencia con características del lenguaje científico, así: preguntas literales concernientes a la precisión, Inferenciales referentes a la concisión, evaluativas para determinar la universalidad y creativas con el fin de establecer la construcción de significados.

\section{Materiales y Métodos}

Una vez realizada la revisión bibliográfica, se establece que este proyecto didáctico pertenece a un tipo de investigación cualitativa, no tradicional que según Cerda (1993), se caracteriza por los siguientes aspectos:

* La interpretación que se da a las cosas y fenómenos que no pueden ser captados o expresados plenamente por la estadística o las matemáticas.

* Utiliza preferentemente la inferencia inductiva y el análisis diacrónico de los datos.

* Utiliza los criterios de credibilidad, transferibilidad y confirmabilidad como forma de hacer creíbles y confiables los resultados de un estudio.

* Utiliza múltiples fuentes, métodos e investigadores para estudiar un solo problema o tema los cuales convergen en torno a un punto central de estudio.

* Utiliza preferentemente la observación y la entrevista abierta y no estandarizada como técnicas en la recolección de los datos.

* Centra el análisis en la descripción de los fenómenos y cosas observadas.

Por otra parte, la propuesta corresponde a una investigación participativa, descrita como una actividad integrada que combina la investigación social, el trabajo educativo y la acción. La meta final es la transformación estructural y el mejoramiento del nivel de vida de la población. Ésta se realiza sobre una comunidad, permitiendo el análisis de una problemática en particular que para el presente proyecto son los aspectos que influyen en el uso del lenguaje científico de un grupo de estudiantes de Biología de grado séptimo en un colegio de la localidad de Ciudad Bolívar. 
Con relación al proceso de interpretación, para Erickson (1986), citado por Stake (1999), es la función que le permite al investigador recoger datos e interpretarlos de manera fundamentada. La principal característica de la investigación cualitativa precisamente es el lugar central que ocupa la interpretación. La cual requiere evitar la emisión de juicios subjetivos, para no perder el contacto con el desarrollo de acontecimientos y con aquello que se va revelando, en parte, para reorientar las observaciones y proseguir con los temas que afloren. Se pueden tomar diferentes caminos para hallar los significados de los casos, para no ir más lejos, la interpretación directa de ejemplos individuales y la suma de los mismos hasta que se pueda decir algo sobre ellos.

Se seleccionaron diferentes fuentes para la recolección de la información, como por ejemplo la observación participante, el diario de clase y la guía de trabajo diagnóstica.

\section{Observación Participante}

Ésta es la técnica clásica primaria y más usada por los investigadores cualitativos para adquirir información. Para ello, el investigador interacciona con las personas o grupos que desea investigar, compartiendo sus usos, costumbres, estilo y modalidades de vida. Al participar en sus actividades corrientes y cotidianas, va tomando notas de campo pormenorizadas en el lugar de los hechos o tan pronto como le sea posible. Estas notas son, después, revisadas periódicamente con el fin de completarlas y, también, para reorientar la observación e investigación respondiendo a las preguntas de quién, qué, dónde, cuándo, cómo y por qué alguien hizo algo, es decir, se consideran importantes los detalles (Martínez, 2006). Además, Kawulich (2005) argumenta que la observación participante recoge datos de procesos.

A partir de la cotidianidad en el aula y las rutinas establecidas, se adoptó la observación participante como una pieza importante del proceso de investigación, de modo que, el criterio de análisis fue la forma cómo los estudiantes usaban el lenguaje para evidenciar los niveles de comprensión de las ideas científicas. La recopilación de los datos se hizo en el diario de clase.

\section{Diario de Clase}

Un instrumento de la observación participante es el diario de clase, en éste, se apuntaron las características más relevantes de la dinámica dentro del aula. Paralelo al desarrollo de las explicaciones, se hicieron las observaciones acerca del lenguaje empleado por los estudiantes, a la vez que, se evaluaron ideas previas, se expuso la temática y se solucionó la guía de trabajo diagnóstica.

En resumen, el diario propicia, el desarrollo de la capacidad descriptiva de la dinámica del aula, por parte de su autor, a través del relato sistemático y pormenorizado de los distintos acontecimientos y situaciones cotidianas.

En esta investigación se tuvieron en cuenta las siguientes recomendaciones dadas por Toscano (1994).

1. Descripción general de la dinámica de clase: organización y distribución de la 
Bio-grafia: Escritos sobre la Biologia y su Enseñanza Vol. 4 No 7. ISSN 2027-1034. Segundo Semestre de 2011P. p. 36-55.

jornada.

2. Descripción pormenorizada de una o varias actividades (las más significativas).

3. ¿Qué hace el profesor durante su desarrollo?

4. ¿Qué hacen los alumnos?

5. Acontecimientos más significativos durante su desarrollo: tipo de conductas, frases textuales (de profesores y alumnos).

6. Descripción de conflictos (si los hubo) entre alumnos y profesor.

7. Dudas, contradicciones, reflexiones que surgen durante o después del desarrollo de las actividades.

\section{Guía de Trabajo Diagnóstica}

Como instrumento de investigación se utilizó la guía de trabajo diagnóstica la cual se diseñó con la intención de medir algunas habilidades cognitivas. Para esto, se tuvieron en cuenta los criterios institucionales para la elaboración de guías, los contenidos de la asignatura y los fines a alcanzar en de la clase de Biología. Esta estructura evalúa fortalezas o debilidades para mejorar un desempeño, que en la presente investigación corresponde al aprendizaje de la Biología, particularmente al uso apropiado de su lenguaje.

Según los autores anteriores, el Dominio dado a la prueba diagnóstica es cognitivo, ya que enfatiza en la comprensión, retención y desarrollo de conocimiento e intelecto. Bajo el mismo esquema, el Tipo de Preguntas elaboradas son clasificadas como de completar, las cuales cumplen con el propósito de consumar frases donde falten palabras 0 conceptos. Dentro de las ventajas ofrecidas por este tipo de preguntas está el hecho de que evalúan respuestas precisas.

Otro de los beneficios de la guía de trabajo diagnóstica, es servir como orientación al estudiante, pues incluye la información necesaria para el correcto uso y manejo provechoso de las actividades de la asignatura. Además de apoyar orientar y conducir el trabajo de la clase.

La guía de trabajo que se diseñó en esta investigación, tiene en cuenta los criterios anteriores y además corresponde a una modificación de Sardá, et.al., (2006) quienes proponen cuestionarios para evaluar cuatro tipos de preguntas, con el propósito de promover distintos tipos niveles de lectura de los textos científicos, partiendo del supuesto de que el tipo de cuestionario puede ayudar a los estudiantes a desarrollar estrategias lectoras. 


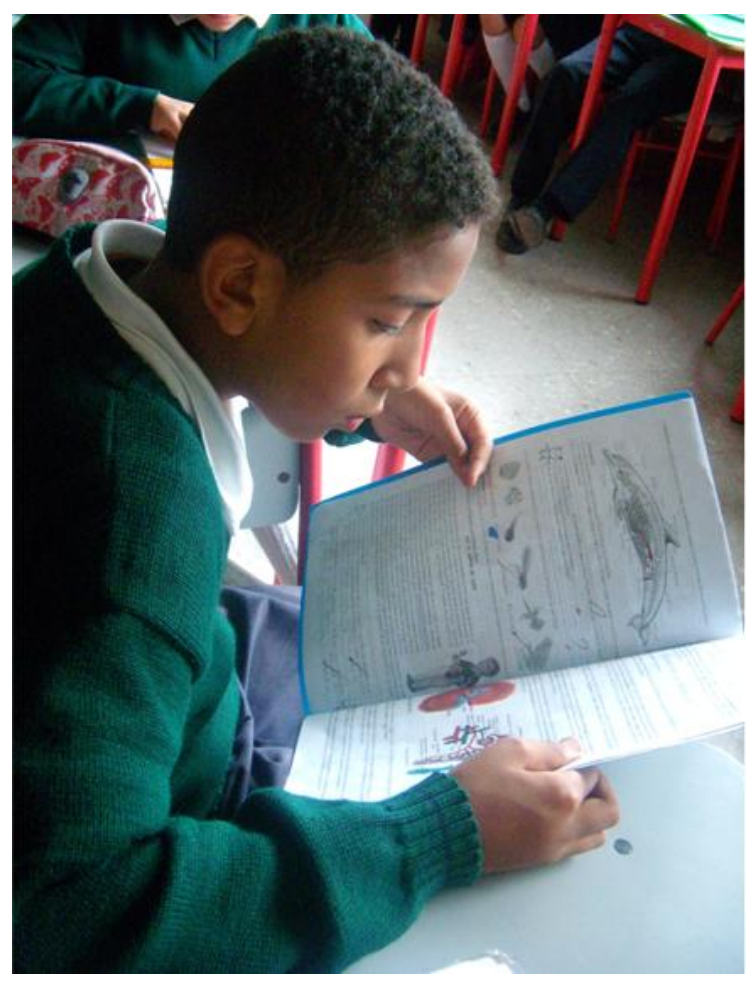

Figura №1. Estudiante de Grado Séptimo D Leyendo Guía de Trabajo Diagnóstica. Tomada por: Erika Lasprilla

A continuación, se describen las características del tipo de preguntas utilizadas.

\begin{tabular}{|l|l||l||l||l||}
\hline Tipo de & Literal & Inferencial & Evaluativa & Creativa \\
\hline \hline \multirow{2}{\text{Pregunta}}{\begin{tabular}{l|l} 
Descripción \\
del análisis
\end{tabular}} & $\begin{array}{l}\text { ¿Qué dice } \\
\text { la Guía? }\end{array}$ & $\begin{array}{l}\text { informaciones no } \\
\text { dice la guía, pero } \\
\text { necesito saber } \\
\text { para entenderla? }\end{array}$ & $\begin{array}{l}\text { ¿Qué valoración } \\
\text { hago de las } \\
\text { ideas de la } \\
\text { guía? }\end{array}$ & $\begin{array}{l}\text { ¿Para qué } \\
\text { me sirve la } \\
\text { guía? }\end{array}$ \\
\hline
\end{tabular}

Tabla №1. Preguntas Literales, Inferenciales, Evaluativas y Creativas

Las respuestas dadas a cada uno de estos tipos de preguntas están relacionadas con la justificación y argumentación de los términos científicos, específicamente respecto al vocabulario empleado sobre la excreción animal. De esta manera, se determinó el grado de comprensión de los estudiantes y su capacidad para adquirir nuevos conocimientos en Biología.

\section{Fases de Investigación}

El proyecto incluye tres grandes fases de trabajo: Fase inicial, Fase de desarrollo y Fase de finalización. Cada una de éstas se define en seguida. 
FASE INICIAL: Entendida a partir de la selección del problema de investigación, la determinación de la población de estudio y el diseño de los enfoques teórico y metodológico.

FASE DE DESARROLLO: Se compone de la planificación, acción y observación para la caracterización del uso del lenguaje científico.

* Planificación: el objetivo de este procedimiento fue la adopción y la elaboración de los instrumentos de investigación para evaluar el grado de apropiación del conocimiento científico por parte de los estudiantes con respecto a la excreción.

* Acción: consistió en la descripción de los aspectos más relevantes consignados en el diario de clase y la aplicación de la guía de trabajo diagnóstica durante las clases de Biología.

* Observación: se realizó de forma reflexiva, analizando e interpretando los resultados con el fin de encontrar las conclusiones de los mismos.

FASE DE FINALIZACIÓN: Radicó en la síntesis de los resultados, cotejados a partir de tres puntos de referencia: las preguntas planteadas en la guía de trabajo diagnóstica, el diario de clase y la revisión de antecedentes lo cual significa que se adoptó un modelo de triangulación para ejercer mayor control sobre las variables consideradas en los resultados.

\section{Categorías de Análisis}

Para Bardin (2012), el análisis por categorías es el más antiguo y el más utilizado, por su parte, Martínez (2006), expone que categorizar es clasificar, conceptualizar o codificar mediante un término o expresión breve que sean claros e inequívocos, el contenido o idea central de cada unidad temática. Las categorías de análisis que conceptualizan la realidad emergen del estudio de la información que se recoja durante los procesos de "contrastación" y de "teorización", es decir, cuando éstas se analicen, relacionen, comparen y contrasten entre sí. Concluyendo entonces que, en el proceso de categorización se va integrando y reintegrando constantemente el todo y las partes, a medida que se revisa el material y va emergiendo el significado de cada sector, evento, hecho o dato.

Por consiguiente, las categorías de análisis para este proyecto se determinaron de acuerdo con el problema de investigación "el lenguaje científico", por lo cual fueron consideradas cuatro de sus características más significativas las cuales se describen en la Tabla $\mathrm{N}^{\circ} 2$. 
Bio-grafia: Escritos sobre la Biologia y su Enseñanza Vol. 4 No 7. ISSN 2027-1034. Segundo Semestre de 2011P. p. 36-55.

\begin{tabular}{|c|c|}
\hline CATEGORÍA I: PRECISIÓN & CATEGORÍA II: CONCISIÓN \\
\hline $\begin{array}{l}\text { Según Amado (2003), es descrita como la } \\
\text { correspondencia biunívoca entre términos y } \\
\text { conceptos. } \\
\text { Tiene correspondencia directa con la } \\
\text { literalidad. }\end{array}$ & $\begin{array}{l}\text { Consiste en la expresión de las ideas con el } \\
\text { menor número de palabras posible como lo } \\
\text { explica Amado (2003). } \\
\text { Tiene correspondencia directa con la inferencia. }\end{array}$ \\
\hline CATEGORÍA III: UNIVERSALIDAD & $\begin{array}{l}\text { CATEGORÍA VI: RECURSOS PARA LA } \\
\text { CONSTRUCCIÓN DE SIGNIFICADOS }\end{array}$ \\
\hline $\begin{array}{l}\text { Para Amado (2003) son los términos que se } \\
\text { manejan ampliamente por toda la comunidad } \\
\text { científica. } \\
\text { Tiene correspondencia directa con la } \\
\text { evaluación. }\end{array}$ & $\begin{array}{l}\text { Finalmente, para Lemke (1997), el lenguaje } \\
\text { científico no es sólo vocabulario y gramática, sino } \\
\text { un sistema de recursos para construir los } \\
\text { significados. } \\
\text { Tiene correspondencia directa con la creatividad. }\end{array}$ \\
\hline
\end{tabular}

Tabla N². Categorías de Análisis

En conclusión, el proceso de categorización, análisis e interpretación, estuvo guiado fundamentalmente por conceptos e hipótesis provenientes o emergentes de la información obtenida y de su contexto propio, que muy bien pudieran ser únicos. En este sentido, para Martínez (2006), la comparación y contrastación pudieran llevar hacia la reformulación, reestructuración, ampliación o corrección de construcciones teóricas previas, logrando un avance significativo en el área.

\section{Triangulación de Datos}

Según Okuda y Gómez (2005), la triangulación se refiere al uso de varios métodos, de fuentes, de datos, de teorías o de ambientes en el estudio de un fenómeno. El término triangulación representa el objeto del investigador en la búsqueda de patrones de convergencia para poder corroborar la interpretación de un objeto de investigación, lo cual no implica que literalmente se tengan que utilizar tres perspectivas. Dentro del marco de la investigación cualitativa, la triangulación supone que al adoptar varias estrategias, las debilidades de cada una no se sobreponen con las de otras y que en cambio sus fortalezas si se suman, pues el considerar solamente una táctica supondría mayor vulnerabilidad a los sesgos y fallas metodológicas, mientras que, tomar en cuenta más referencias posibilita el aumento de la validez y consistencia de los hallazgos.

Para Stake (1999), la triangulación es una estrategia a través de la cual se halla la precisión de las explicaciones dejando de lado la simple intuición y las buenas intenciones de "hacerlo bien". Es decir, que es un método para saber si aquello que se observa tiene el mismo significado cuando se encuentra en otras circunstancias, lo cual obliga una vez y otra vez a la revisión. 
Para caracterizar e interpretar el uso del lenguaje científico en los estudiantes de grado séptimo sobre la excreción, en este proyecto se analizaron los resultados, cotejándolos a partir de tres puntos de referencia: las preguntas planteadas en la guía de trabajo diagnóstica, el diario de clase y la revisión bibliográfica de antecedentes. Por esta razón, se establece una valoración de los resultados obtenidos a partir de tres entes "los estudiantes de grado séptimo", "la docente de Biología" y "los autores de los documentos de referencia". Por lo tanto, se resalta la importancia del diálogo con los investigadores que precedieron el estudio, para corregir, mejorar, ampliar o reformular conclusiones, es decir, para enfocarlas desde otros puntos de vista y con el uso de otras categorías, con lo cual se profundizó en la comprensión del objeto de estudio.

\section{Población de Estudio}

Al momento de realizar una clase y más de analizar algunos de sus aspectos es necesario tener en cuenta los contextos en los cuales se enmarca la práctica educativa, ya que éstos inciden en la forma cómo se establece la relación entre estudiantes estudiante, maestro - estudiante, maestro -conocimiento y estudiante - conocimiento. Dependiendo de las variables que se presenten en el contexto educativo incluyendo familia, barrio, estrato socioeconómico, nivel de estudio, entre otros, el maestro y el estudiante van a asumir determinados roles y conductas que permean la dinámica del aula, facilitando o dificultando el proceso de enseñanza - aprendizaje. Debido a esto, lo educativo no se puede sustraer del sistema sociocultural en el cual se encuentra inmerso.

El contexto donde se realiza la investigación es el Centro Educativo Distrital Calasanz, un colegio en concesión con la Secretaría de Educación y la Fundación Social Calasanz, ubicado en el barrio Buenos Aires -Ciudad Bolívar-. Durante un periodo de tiempo comprendido entre los meses de Abril y Mayo de 2010, con el grado Séptimo D se aplicaron las guías diagnósticas en las clases de Biología sobre la excreción animal. La muestra incluye adolescentes cuyas edades oscilan entre los once y catorce años pertenecientes a los estratos uno y dos, según se sabe a nivel institucional.

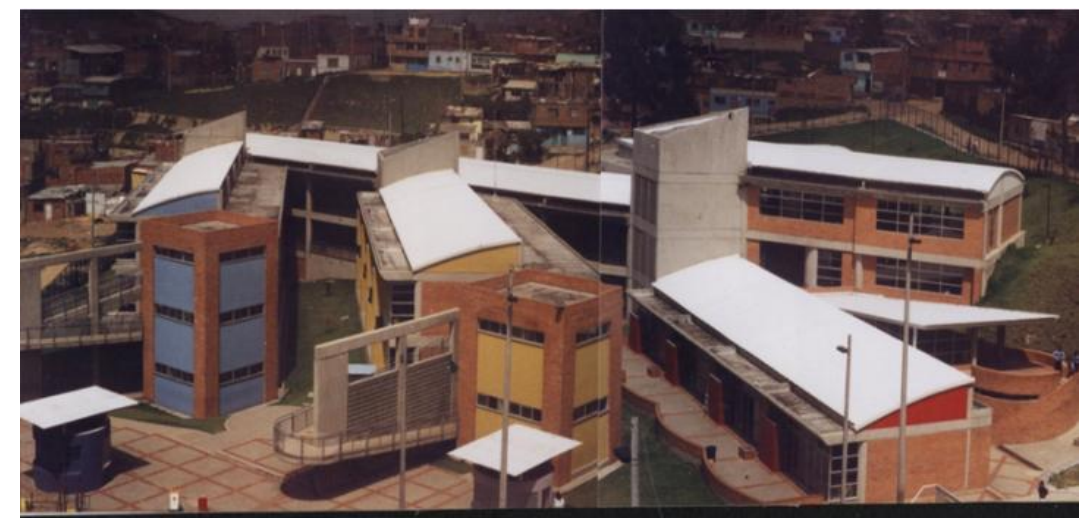

Figura №2. Panorámica del Centro Educativo Distrital Calasanz Ubicado en el Barrio Buenos Aires Tomada por: Institución.

La localidad de Ciudad Bolívar se ubica en el sur de Bogotá D.C. Comprende los siguientes límites: al Norte, con la localidad de Bosa, al Sur, con la localidad de Usme al 
Oriente con la localidad de Tunjuelito y Usme, y al Occidente, con el municipio de Soacha. Ciudad Bolívar es la tercera localidad más extensa de Bogotá y la segunda con mayor índice de necesidades básicas insatisfechas y déficit en servicios públicos. Predomina la tendencia al desarrollo de asentamientos ilegales por personas del área rural y de provincia. Esta compleja articulación de una dinámica ilegal de ocupación del territorio, condiciones sociales y económicas de pobreza, deficiente infraestructura, carencia de equipamientos y presencia de múltiples formas delictivas, convierte a la localidad en escenario de múltiples situaciones que afectan la convivencia de sus habitantes. También es importante resaltar que según información del Departamento Administrativo de Planeación Distrital, esta zona se encuentra dentro de las localidades con mayor número de la Población en Edad Escolar del Distrito Capital, concentrando en el 2002 el 10,7\% (164.200 niños y jóvenes) de la PEE del Distrito que asciende a 1.540.798 habitantes.

A pesar de esta realidad social de la localidad no se puede ignorar el hecho de que, tanto la Alcaldía de Bogotá, como entidades privadas vienen haciendo esfuerzo por ofrecer a sus pobladores posibilidades de desarrollo tanto en lo humano como en sus necesidades básicas. Una de estas organizaciones es la Fundación Social Calasanz, la cual brinda posibilidades de una educación de calidad para los niños de escasos recursos. Así pues, el CED Calasanz lleva ese nombre en honor a José de Calasanz fundador de la orden religiosa de los padres escolapios y de las escuelas pías, también creador de la escuela popular cristiana la cual comenzó hace más de 400 años en Roma, con el propósito de educar a los niños más pobres.

La población es el conjunto de elementos que forma parte de un grupo de estudio, es decir, el objetivo o propósito central. Según lo anterior, la población de estudio para este proyecto fue el grado Séptimo D, conformado por 43 estudiantes del Centro Educativo Distrital Calasanz en Ciudad Bolívar. Para Martínez (2006), la elección se hace de forma intencional por medio de una serie de criterios que se consideran necesarios o altamente convenientes para tener una unidad de análisis con las mayores ventajas hacia los fines que persigue la investigación.

\section{Resultados y discusión}

Considerando la estructura metodológica planteada en esta investigación, se presentan los siguientes resultados. Inicialmente, se analiza la población de estudio, luego, se presenta la evaluación de ideas previas y más adelante, se exponen las respuestas de los estudiantes frente a las preguntas propuestas en la guía de trabajo diagnóstica desde las cuales se determinó la capacidad de comprensión y expresión del lenguaje científico.

\section{Características Socio Económicas y Culturales de la Población de Estudio}

A partir de la revisión documental, se encontró un estudio realizado por Aja (2009), donde se muestran determinados rasgos socioeconómicos de los niños y niños de bachillerato en el Ced Calasanz, de esta manera, en la Figura $N^{\circ} 3$ se mencionan las características correspondientes al grado séptimo. 


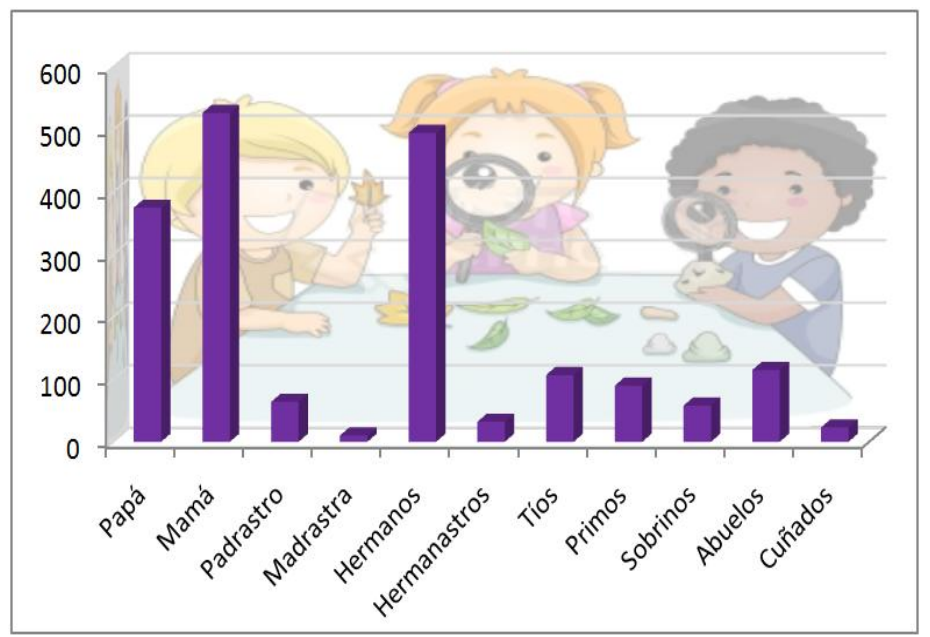

Figura $\mathbf{N}^{\circ} \mathbf{3}$. Conformación del Núcleo Familiar de los Estudiantes

* La edad en la cual se encuentran los estudiantes está comprendida entre 11 y 14 años.

* Los estudiantes pertenecen a los estratos 1 y 2.

* La mayoría conviven con su madre, seguido por el porcentaje que vive con sus hermanos y por último, los que conviven con su padre. En términos de porcentaje coincide con los datos suministrados por el censo del 2005, donde se indica que uno de cada tres niños que nacen en Colombia carecen de padre responsable.

* Un alto porcentaje vive en casa de propia y algunos viven en arriendo o con otros familiares como pueden ser tíos o abuelos. Por lo cual, se puede inferir que aquellos que poseen casa propia son los padres que llevan más años de relación.

Otras cuestiones son descritas en documentos de trabajo elaborados por el Departamento de Ciencias Sociales de la institución (sin publicar). A partir del análisis de estos docentes se obtiene que:

* El barrio al cual pertenecen la mayoría de los estudiantes es el Buenos aires, donde se encuentra ubicada la institución, seguido del Diamante, las Quintas, San Joaquín, Divino Niño, Lucero y otros más lejanos a la zona como: Monterrey, Villa Dana, Diana Turbay, Arabia, San Francisco, Vista Hermosa y Kennedy.

* La estructura familiar está dada en su mayoría por padres casados seguidos de la unión libre.

Para finalizar, se les preguntó a los estudiantes, cuál era el grado de escolaridad de sus padres y los resultados obtenidos se muestran en la Tabla:

\begin{tabular}{|l||l||l||}
\hline NIVEL EDUCATIVO & PADRE & MADRE \\
\hline \hline No Posee Ningún Tipo de Estudios & 1 & 0 \\
\hline \hline Estudios Primarios Incompletos & 4 & 5 \\
\hline
\end{tabular}


Bio-grafia: Escritos sobre la Biologia y su Enseñanza Vol. 4 No 7. ISSN 2027-1034. Segundo Semestre de 2011P. p. 36-55.

\begin{tabular}{||l||l|l|}
\hline Estudios de Primaria Completa & 7 & 6 \\
\hline \hline Estudios de Bachillerato Incompletos & 9 & 15 \\
\hline \hline Bachiller & 9 & 10 \\
\hline \hline Técnico o Tecnólogo & 1 & 1 \\
\hline \hline Profesional & 0 & 4 \\
\hline \hline
\end{tabular}

Tabla №2. Nivel Educativo de Padres y madres de los Estudiantes de Grado Séptimo D del Ced Calasanz

\section{Lenguaje Científico: Guía de Trabajo Diagnóstica}

Durante seis sesiones los estudiantes de Séptimo D solucionaron la guía de trabajo diagnóstica de forma alterna con el desarrollo normal de una clase de Biología, en este mismo espacio, se consignaron las observaciones del diario. A continuación, se muestran las respuestas obtenidas en cada una de las preguntas sobre la excreción animal con las que se caracterizó el uso del lenguaje científico.

\section{RESPUESTAS A LAS PREGUNTAS DE TIPO LITERAL}

Las preguntas de este tipo evalúan el contenido textual de la información presentada en la guía de trabajo. En la siguiente gráfica se resumen las respuestas de los alumnos, se consideran como correctas aquellas que utilizan precisión en su lenguaje, es decir, por la correspondencia biunívoca entre términos y conceptos (Amado, 2003).

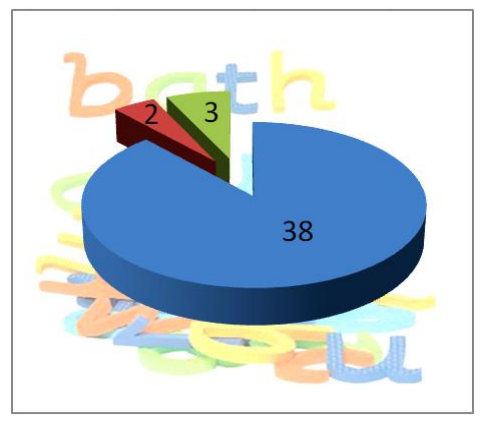

1. ¿Cuáles son las sustancias que se producen durante la excreción?

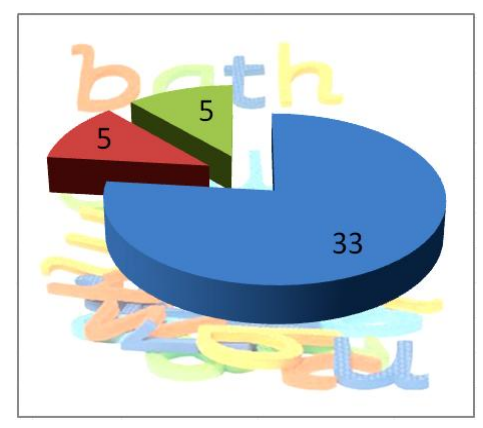

2. ¿Qué es excreción?

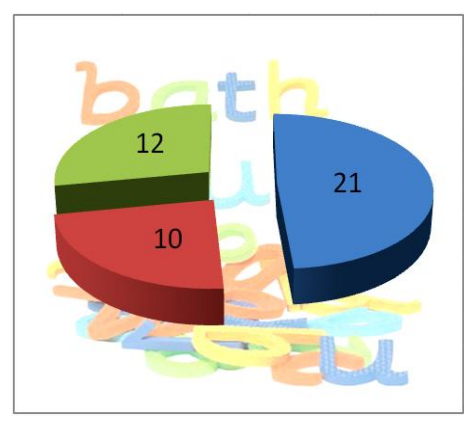

3. ¿Cuáles son las estructuras de excreción de los invertebrados?

- Respuestas Correctas $\square$ Respuestas Incorrectas

Respuestas en Blanco o Incompletas

Figura N4. Respuesta a las Preguntas de Tipo Literal

Según se observa en la Figura $\mathrm{N}^{\circ} 4$, las preguntas uno y dos cuyas respuestas son cortas, fueron respondidas correctamente por la mayoría del grupo. Sin embargo, el interrogante tres que requería de argumentos más extensos produjo dificultad para dar cuenta de la comprensión del tema a tratar.

Para Sardá, et.al. (2006), las respuestas a las preguntas literales exhiben el pragmatismo propio de la adolescencia, el cual conduce a los alumnos a responder utilizando solamente copias parciales de la información del texto como se mostró en los resultados de este proyecto.

De la totalidad de la población, 38 estudiantes están en capacidad de reconocer el dióxido de carbono, las sales minerales, los compuestos nitrogenados: amoniaco, urea y ácido 
úrico como las sustancias resultantes de la excreción. Mientras que, en el interrogante $N^{\circ} 2$, se indica que 10 alumnos no dan cuenta con precisión de qué es la excreción.

La pregunta concerniente a las sustancias de excreción propias de invertebrados causó la mayor dificultad, no por desconocimiento de las mismas, sino por el bajo dominio de los nombres de los grupos invertebrados como se anota en el diario de clase.

RESPUESTAS A LAS PREGUNTAS DE TIPO INFERENCIAL

Las inferencias se refieren a la información que no es suministrada por la guía, pero, que un estudiante debe conocer para entender la pregunta planteada. Como correctas fueron consideradas las respuestas concisas, entendiendo la concisión como una de las características del lenguaje científico que posibilita la expresión de ideas usando el menor número de palabras posible (Amado, 2003).

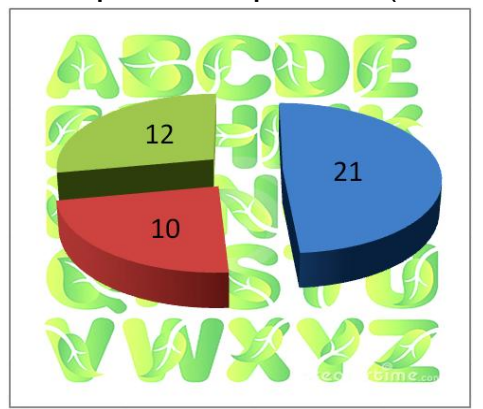

1. Si el cuerpo de Juan se hincha por aumento de sales en su sangre a causa de una deficiencia en el funcionamiento de su riñón, ¿de qué manera puede controlar la hinchazón de su cuerpo mientras el riñón vuelve a funcionar normalmente?

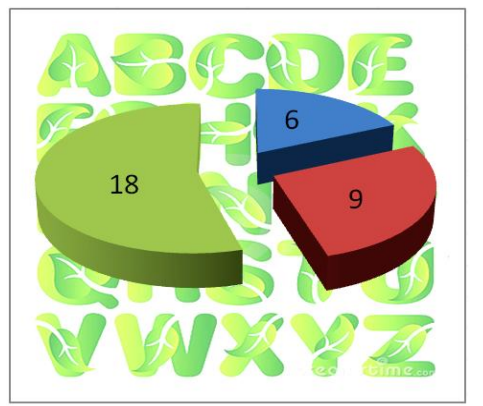

3. Si tú fueras un ser acuático, ¿cuáles serían las características de tu sistema excretor?

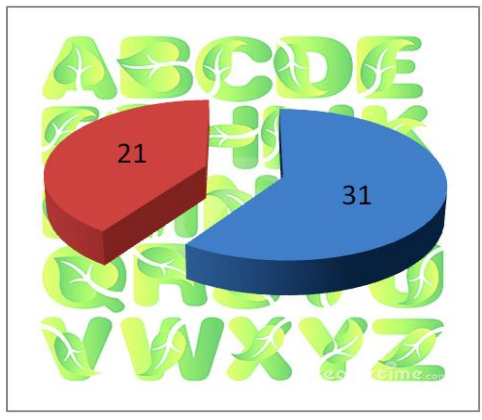

2. ¿Qué podría sucederle a un ser si deja de excretar?

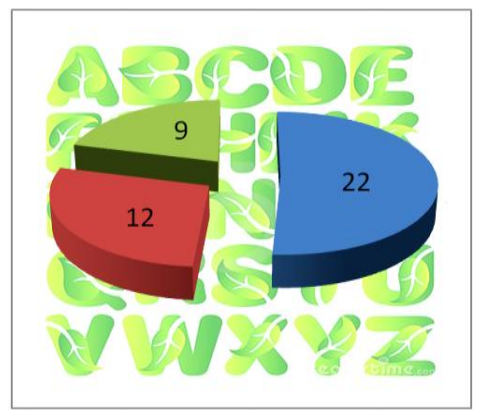

4. ¿Qué ocurriría en el riñón si se aumenta la cantidad de nefronas?

\section{- Respuestas Correctas $\quad$ Respuestas Incorrectas}

\section{Respuestas en Blanco o Incompletas}

Figura $\mathbf{N}^{\circ}$ 5. Respuesta a las Preguntas de Tipo Inferencial

Algunos referentes señalan que el alumno desde la primaria se encuentra en capacidad de realizar inferencias, más los resultados de la Figura $N^{\circ} 5$, indican que el nivel Inferencial de las respuestas es bajo, en conjunto incorrectas y en blanco corresponden a la mayoría. En el estudio de Sardá, et. al. (2006), se encuentra que cuando los estudiantes responden preguntas de este tipo deben evitar el uso de estrategias reproductivas, haciéndose necesario el razonamiento. Lo anterior, lleva a concluir que no se tiene manejo de concisión del lenguaje formal, por lo tanto, se asume que las preguntas de inferencia causan mayor dificultad que las literales.

La mitad de los educandos carecen de la habilidad para explicar cuando se les pregunta qué puede sucederle a un ser si deja de excretar. Mientras que, si se les interroga de otra 
forma, como en el caso de la pregunta literal: ¿qué es la excreción? saben dar respuestas correctas. Por otro lado, en la pregunta evaluativa sobre las funciones de la excreción, la mayoría pudo dar una respuesta satisfactoria como se explica más adelante.

\section{RESPUESTAS A LAS PREGUNTAS DE TIPO EVALUATIVO}

Las preguntas evaluativas se refieren a la valoración que se hace de las ideas. La característica del lenguaje que se estimó en esta oportunidad es la universalidad, la cual implica un manejo de los términos por parte de la comunidad científica (Amado, 2003).

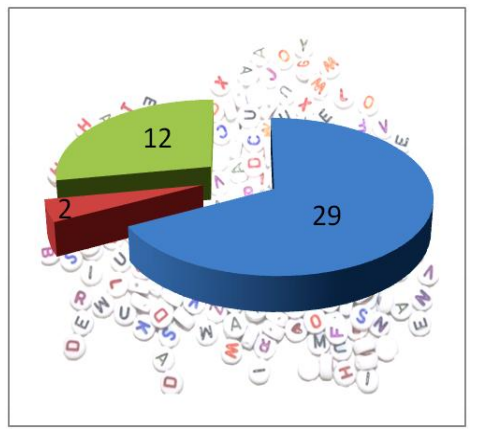

1. Anota tres de las principales funciones del riñón

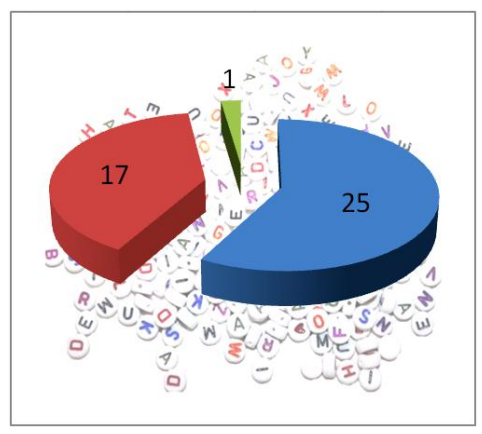

3. ¿Por qué los animales acuáticos y terrestres tienen diferentes formas de excreción?

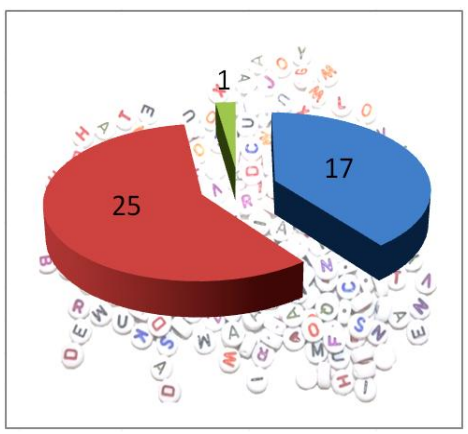

2. ¿Cuál es la idea principal de los textos?

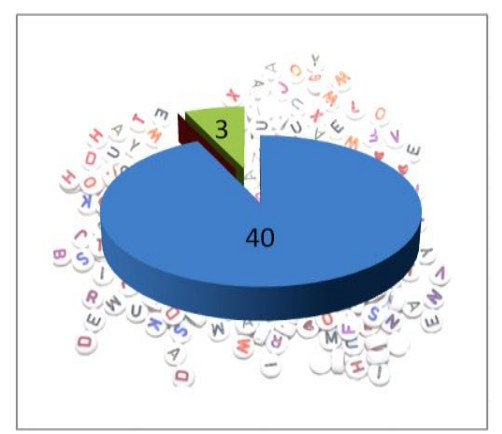

4. Tomás desea saber, ¿cuál de los siguientes volúmenes es necesario para producir la micción?

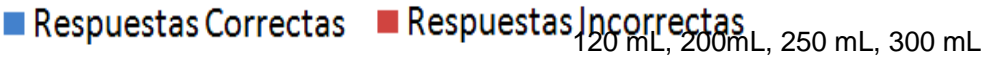 Respuestas en Blanco o Incompletas}

Figura N6. Respuesta a las Preguntas de Tipo Evaluativo

La forma en la cual se presentan las respuestas varía entre la pregunta uno a cuatro (Figura $\mathrm{N}^{\circ} 6$ ), podría pensarse entonces que, los estudiantes comprenden las funciones del riñón y lo expresan con términos "universales" mientras que, hay dificultades en el dominio de la cantidad de términos asociados con la excreción del resto de los animales que conforman el reino.

Las preguntas de carácter evaluativas tuvieron la mayoría de respuestas correctas como se observa más adelante en la Figura N8. Ningún estudiante dejó de responder la pregunta: ¿cuál es el volumen necesario para producir la micción?, además, porque se trata de la única que posee opciones de respuesta.

Por otra parte, un poco más de la mitad de los estudiantes diferencia las características de excreción de los animales acuáticos y los terrestres. De nuevo se muestra que hay una 
comprensión textual sesgada, pues la mayoría no supo dar cuenta de la idea principal de la guía de trabajo diagnóstica.

Sardá, et.al., (2006), categorizan las preguntas evaluativas como de difícil comprensión porque requieren la asimilación global del texto y la confrontación de la información obtenida y la que se tiene, sin embargo, y según los resultados observados, en la Figura N8, se ve cómo fueron las preguntas con mejor resultado.

\section{RESPUESTAS A LAS PREGUNTAS DE TIPO CREATIVO}

Este estilo de preguntas se representa en razón a ¿para qué me sirve la guía? ¿Estas ideas pueden ser útiles en la interpretación de otros fenómenos? Son establecidas como respuestas correctas aquellas que cumplen con la propuesta de Lemke (1997), respecto a que, el lenguaje científico no sólo es vocabulario y gramática: es un sistema de recursos para construir significados.

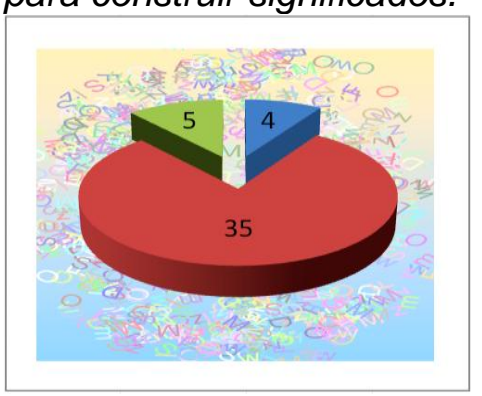

1. Susanita es una rana, en un día, puede perder en agua un tercio del peso de su cuerpo a través de la piel y la orina. Si hoy pesa $300 \mathrm{~g}$, ¿cuánto pesará Susapita mañana después de la excreción?

\section{Respuestas en Blanco o Incompletas}

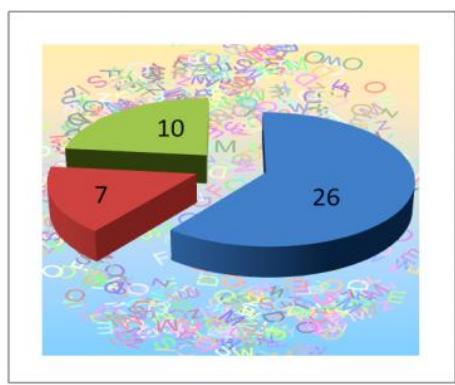

3. ¿Cómo colectarías las sustancias de excreción de un animal invertebrado? ¿De cuál animal en particular?

Figura N7. Respuesta a las Preguntas de Tipo Creativo

En la Figura $\mathrm{N}^{\circ} 8$ se aprecia que una pregunta en particular causó gran dificultad. A pesar de esto, en su totalidad los interrogantes de tipo creativo son comprendidos por la mayoría de la población de estudio. Las inferencias y las preguntas creativas causan mayor incomprensión que las literales y evaluativas, puesto que, las primeras se relacionan en cuanto a su estructura gramatical. 


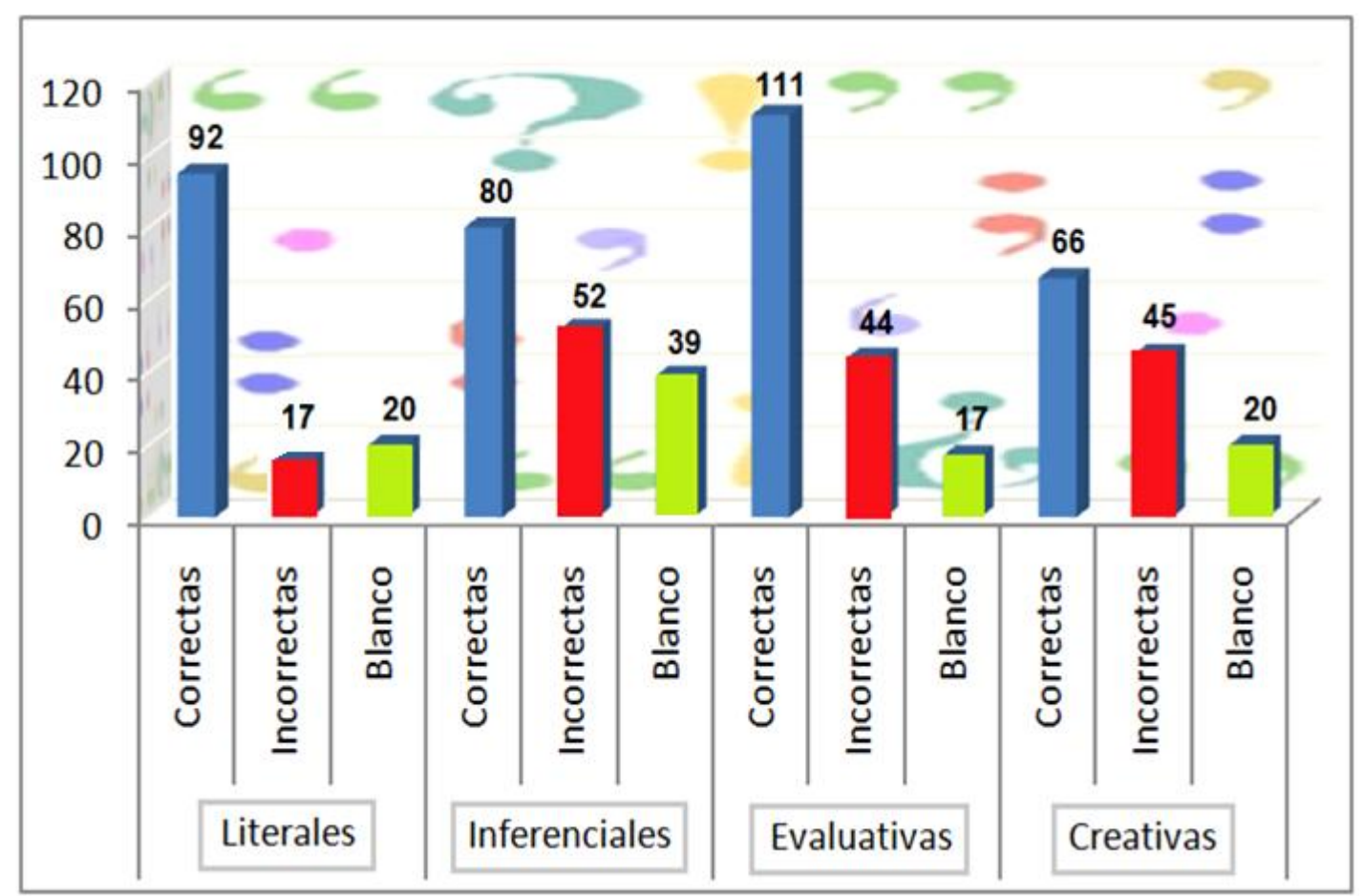

En resumen...

Figura N8. Consolidado de Respuestas e Incorrectas

Con respecto a la universalidad en el lenguaje, característica que fue estimada por medio de las preguntas de tipo evaluativo, se observan los mejores resultados en la población de estudio (Figura $N^{\circ} 4$ ). De forma particular, hay comprensión de las funciones del riñón y las diferencias entre la excreción de animales acuáticos y terrestres. En su mayoría, los estudiantes reconocen lo que Amado (2003), describe como los términos manejados ampliamente por la comunidad científica a nivel global. En relación con las preguntas de estilo inferencial, son las que produjeron mayor número de dificultades en los estudiantes. No hay concisión para dar explicación acerca de la eficacia de las nefronas y de qué sucedería si se presenta un desequilibrio en la excreción del hombre (Figura N5).

Después de las preguntas evaluativas, los alumnos están en capacidad de dar respuesta a las de tipo literal, resultado coincidente con la investigación de Sardá, et.al (2006), quienes argumentan que la mayoría de los cuestionarios en los documentos de ciencias promueven la lectura literal, la desventaja reside en que en ocasiones impide el establecimiento de relaciones entre los conocimientos de los textos y los propios.

El estilo de preguntas creativas no posibilitó a la población la construcción de significados con sentido, como es característico del lenguaje formal. Los estudiantes exhiben dificultad al dar cuenta de la contextualización de la información, por ejemplo, no identifican de qué manera recuperan líquidos los anfibios después de excretar, aunque están en capacidad de hablar empleando algunos términos de la excreción, no los pueden explicar en situaciones reales.

\section{Conclusiones}

* El papel del lenguaje científico es preponderante en la comprensión de los procesos biológicos. Se determina que los estudiantes con mayor habilidad en el manejo de conceptos se desenvuelven mejor en clase. De esta manera, propender por la adquisición del lenguaje científico posibilita mejorar procesos de enseñanza - aprendizaje y transformar las realidades escolares que viven los niños y niñas en las instituciones educativas pertenecientes a estratos bajos, como por ejemplo, el Centro Educativo Distrital Calasanz de la localidad de Ciudad Bolívar. 
Bio-grafia: Escritos sobre la Biologia y su Enseñanza Vol. 4 No 7. ISSN 2027-1034. Segundo Semestre de 2011P. p. 36-55.

* Por medio de la guía de trabajo diagnóstica es posible analizar el nivel de apropiación del lenguaje científico durante las dinámicas normales de las clases. A su vez, la estimación de las características del lenguaje facilita el reconocimiento de fortalezas y debilidades de la comunicación de ideas científicas de los estudiantes. A partir de dicho reconocimiento, es posible proponer estrategias pedagógicas que permitan reducir los errores y potenciar las fortalezas en la comprensión y expresión. Inicialmente, se analiza desde la temática de excreción animal, por la riqueza de su vocabulario, lo cual constituye un punto de referencia para mejorar el aprendizaje de éste y otros procesos biológicos.

* Otra ventaja de la guía de trabajo diagnóstica es su carácter formativo además de evaluativo, lo cual favorece la obtención de datos representativos para el análisis de una situación en particular. Por otra parte, se trata de un instrumento de investigación que permite establecer condiciones para el mejoramiento del proceso estudiado. Con respecto al análisis del lenguaje científico empleado en la comprensión de la excreción animal en adolescentes de grado séptimo D del Ced Calasanz, por medio de este instrumento, se evalúa el nivel de conocimiento, así como, las actitudes y aptitudes de los alumnos, a través de un procedimiento sencillo de evaluación interna.

* La población de estudio pertenece a los estratos uno y dos, lo cual determina sus condiciones de vida y por ende de educación. el núcleo familiar de los estudiantes está conformado por padres con bajo nivel académico, en su mayoría, las madres son jefe de familia y los niños y niñas carecen de suficiente acompañamiento en las actividades escolares, en primer lugar, por la falta de formación del adulto y en segundo lugar, por el escaso tiempo que comparten padres e hijos. Lo anterior, supone una mayor responsabilidad de la escuela en la formación de los estudiantes del Ced Calasanz.

*En cuanto a la comprensión del lenguaje formal, en la población de estudio se encuentra determinada por diversos factores de distinta naturaleza, que influyen en mayor o menor grado en su apropiación. Así, por ejemplo, están los elementos socio culturales y económicos, los intereses del adolescente, las características de la institución educativa, el plan de estudios y las prácticas pedagógicas, entre otras variables que determinan la manera en que el alumno se expresa dentro y fuera de la clase de Biología. Según lo anterior, los estudiantes poseen un nivel de comprensión aceptable respecto a la excreción animal y el léxico que se relaciona con el proceso.

* Se observa que una de las funciones vitales de los organismos como lo es la excreción animal, es entendida por la mayoría de los estudiantes de grado séptimo en un nivel aceptable. Por ende, la relación que existe entre el proceso biológico y el lenguaje científico, está dada por la dificultad en el dominio de los términos relacionados con la excreción y el vocabulario asociado con cada concepto. Otro factor determinante de este suceso, es la falta de lectura comprensiva de los textos y finalmente, las ideas previas que se encuentran arraigadas a la información que posee el alumno.

* Una de las principales causas de dificultad en la comprensión del lenguaje científico es la falta de habilidad lectora de los alumnos. El hecho de que en clase de Biología no se entienda la información suministrada por medio de guías u otros documentos, disminuye la capacidad de aprendizaje de las ciencias. Lo anterior, supone una responsabilidad que involucra al docente, quien debe exhortar y corregir al educando cuando cometa errores en sus expresiones orales o escritas, debe animarlo a expresar adecuadamente los conceptos referentes a la excreción animal $\circ$ a cualquier otra temática que, produzca dificultades similares o mayores a las presentadas con el vocabulario relacionado con dicho fenómeno biológico.

*El estilo de preguntas sobre las cuales los estudiantes de grado séptimo tienen mayor dominio, son las de carácter evaluativo referentes a la universalidad del lenguaje científico, seguidas de las literales correspondientes a la precisión, mientras que, las de inferencia causan mayor dificultad, es decir, las concisas y finalmente las creativas que suponen la construcción de significados. Por 
Bio-grafia: Escritos sobre la Biologia y su Enseñanza Vol. 4 No 7. ISSN 2027-1034. Segundo Semestre de 2011P. p. 36-55.

su parte, los alumnos señalan que las preguntas literales son las que a ellos les parecen más entendibles, debido a que, éstas consisten en repetir la información que se encuentra plasmada en la guía de trabajo diagnóstica lo cual no requiere siempre del uso de habilidades de pensamiento.

*El diseño metodológico adoptado en este proyecto, resultó adecuado tanto para la recolección de la información, como para la interpretación de las variables. Es decir que, a partir de una investigación de tipo cualitativo y de la observación participante, utilizando una guía de trabajo diagnóstica, el diario de clase y la revisión bibliográfica, se llegó a la caracterización del lenguaje científico de los estudiantes de la clase de Biología sobre excreción animal, en el grado Séptimo D del Centro Educativo Distrital Calasanz.

* La escuela desempeña un papel relevante en los procesos de aprendizaje de la Biología en los estudiantes de grado séptimo del Ced Calasanz. Durante la caracterización de la población, se demostró que el núcleo familiar de los escolares está conformado por adultos que en su mayoría carecen de formación profesional, lo cual implica una relación entre la participación de la familia y el rendimiento educativo. Por otra parte, así el adolescente conviva con personas mayores, cuando se trata de un único padre, éste debe dedicarse al sostenimiento del hogar, y en consecuencia, el alumno cuenta únicamente con sus pares y maestros para acompañar los procesos académicos.

\section{Recomendaciones}

*Se recomienda, promover la lectura científica con objetivos definidos, desde la institución, el plan de estudios y el docente, de tal manera, que se convierta en una tarea fundamental que debe ser ejecutada por toda la comunidad educativa. Del mismo modo, como se estimula la lectura, deben generarse estrategias que garanticen que los estudiantes produzcan expresiones orales y escritas con dominio de un léxico científico que le permita: extraer ideas principales, elaborar interpretaciones, relacionar conceptos y emplear el conocimiento adquirido. Los objetivos anteriores se relacionan con los propuestos en la estrategia pedagógica de esta investigación, es decir que, su implementación es un punto de partida.

*Como una de las proyecciones a mediano plazo, se considera fomentar la responsabilidad de la familia en el proceso formativo de sus hijos e hijas, de tal manera que, la institución educativa posibilite de algún modo la alfabetización científica de los adultos para que éstos orienten los procesos académicos de los estudiantes a su cargo. Con lo anterior se espera acrecentar el nivel cultural de las personas habitantes de los estratos bajos como ocurre en la localidad de Ciudad Bolívar.

\section{Referencias Bibliográficas}

AJA, L. (2009). Informe de Resultados: Estudio Epidemiológico con la Población Estudiantil Secundaria del Colegio Calasanz, Ciudad Bolívar. Bogotá D.C: Fundación Buscando Ánimo.

AMADO, J. (2003). El lenguaje científico y la lectura comprensiva en el área de las ciencias. Blitz serie Amarilla. España: Gobierno de Navarra. 61 p.

BARDIN, L (2002). Análisis del Contenido. Madrid: Akal Ediciones. 191 p. 
Bio-grafia: Escritos sobre la Biologia y su Enseñanza Vol. 4 No 7. ISSN 2027-1034. Segundo Semestre de 2011P. p. 36-55.

CERDA, H. (1993). Elementos de la Investigación. Cómo Conocerlos, Diseñarlos y Construirlos. Abya Yala Quito. Edición autorizada por Editorial el Búho. 443 p.

FOUCAULT, M. (1983). El orden del Discurso. Barcelona: Tusquets, Segunda Edición.

KAWULICH, B. (2005). La Observación Participante como Método de Recolección de Datos. Volumen $6(2)$

KEMPA, F. (1991). Students' Learning Difficulties In Science. Causes and Possible Remedies. En: Enseñanza de las Ciencias. Volumen 9 (2). pp. 119 - 128. California.

LEMKE, J. (1997). Aprender a hablar ciencia: Lenguaje, aprendizaje y valores. España: Paidos Ediciones. $273 \mathrm{p}$.

MARTÍNEZ, M. (2006). La Investigación Cualitativa. En: Revista IIPSI. Facultad de Psicología UNMSM. Volumen 9 (1). pp. 123 - 146.

OKUDA, M; GÓMEZ, C. (2005). Métodos de Investigación Cualitativa: Triangulación. En: Revista Colombiana de Psiquiatría. Volumen 34 (1).pp. 118 - 124. Bogotá.

SARDÁ, A; MÁRQUEZ, C; SANMARTÍ, N. (2006). Cómo Promover Distintos Niveles de Lectura de los Textos de Ciencias. En: Revista Electrónica de la Enseñanza de las Ciencias. Volumen 5 (2). pp. $\quad 290-303$.

STAKE, R. (1999). Investigación con Estudio de Casos. Segunda edición. Madrid: Ediciones Morata. $159 \mathrm{p}$.

TOSCANO, J. (1994). Un Recurso para Cambiar la Práctica: El Diario del Profesor. En: Revista Kikiriki Número 33. pp 35-40. IES Bellavista. Sevilla: Grupo DIE. Red IRES. 149 p. 